\title{
Chiropraktik beim Sporthund
}

\author{
Welche Folgen haben Agility, Obedience \& Co.?
}

Johanna Reinhardt

\section{Zusammenfassung}

Hunde werden heute in den unterschiedlichsten Bereichen des Hundesports eingesetzt. Je nach Sportart treten unterschiedliche Belastungen auf, durch die es zu Verletzungen (Zerrungen, Stauchungen, Frakturen) kommen kann. Viel häufiger sind leistungs- und eventuell auch alltagseinschränkende Veränderungen am Bewegungsapparat bzw. einzelnen Strukturen, die eher unauffällig verlaufen und schulmedizinisch häufig nicht greifbar bleiben. Hier können Diagnostik und Therapie durch einen ausgebildeten TierChiropraktiker helfen, den Tieren ihre gewohnte Lebensqualität und Leistungsfähigkeit wieder zurückzugeben.

\section{Was genau ist Chiropraktik?}

Chiropraktik ist eine manuelle Behandlungsmethode. Das Wort Chiropraktik leitet sich aus dem Griechischen ab: „cheiros“ - mit der Hand. Wörtlich bedeutet es also „mit den Händen behandeln“.

Im Zentrum der chiropraktischen Behandlung stehen die gestörte Funktion der Wirbelsäule und die Auswirkungen dieser Störungen auf das Nervensystem und den Gesamtorganismus. Blockaden (vertebrale Subluxationskomplexe) in der Wirbelsäule schränken den normalen Bewegungsspielraum der betreffenden Gelenke ein. Auch die Stukturen, die das Gelenk umgeben (Bänder, Nerven und Muskeln), sind von diesen Bewegungseinschränkungen betroffen. Unwillkürlich versucht das Tier, Körperhaltung und Bewegungen anzupassen und die Bewegungseinschränkung über andere Bewegungseinheiten zu kompensieren. Oft treten dann als Folge Überbeanspruchungen der entsprechenden Strukturen und weitere Blockaden auf. Kompensationsblocka- den entstehen entlang der Wirbelsäule, wodurch die natürliche Biomechanik des Bewegungsapparates gestört wird. Steifheit, Schmerzen und verminderte Leistungsfähigkeit bis hin zu Verschleißerscheinungen sind häufige Folgen dieser funktionalen Störungen.

Da das Rückenmark mit seinen austretenden Spinalnerven innerhalb des Wirbelkanals liegt, verändern bereits kleinste Blockaden den Informationsfluss des Nervensystems und beeinflussen so das Gleichgewicht des gesamten Organismus. Ein professionell ausgebildeter (Tier)Chiropraktiker kann mit speziellen Untersuchungstechniken solche Bewegungseinschränkungen erkennen und gezielt beheben. Mit einem schnellen, kurzen Impuls (Adjustment) innerhalb des physiologischen Bewegungsspielraums in genauem Winkel zu der betreffenden Bewegungseinheit werden die einzelnen Gelenke spezifisch behandelt und die natürliche Beweglichkeit des Gelenks wiederhergestellt. Dies ermöglicht eine Regeneration des Organismus.

\section{Der Sporthund}

Hunde sind heute in verschiedensten Bereichen des Hundesports tätig. Hierbei werden sie den unterschiedlichsten körperlichen Herausforderungen ausgesetzt. Der sportliche Einsatz reicht hier von gelegentlichem Training ohne Wettkampfeinsatz bis hin zu mehrfach wöchentlichem Training und Turnieren an fast jedem Wochenende.

\section{Agility}

Bei der sehr beliebten Sportart Agility geht es darum, den Hund in der korrekten Reihenfolge fehlerfrei durch einen Hindernisparcours (Sprünge, Kontaktzonengeräte, Slalom, Tunnel) zu führen - und das mög- lichst schnell [5]. Viele der Hunde stehen hier unter einem hohen Trieb und absolvieren die Parcours mit einer Geschwindigkeit von weit über $5 \mathrm{~m} / \mathrm{s}$. Dabei werden je nach Gerät und Art der Ausführung unterschiedliche Muskelgruppen und Gelenke der Hunde beansprucht.

Der jeweilige Ausbildungsstand des Teams - sowohl die bereits erworbenen Fähigkeiten des Hundes als auch die führerischen Fähigkeiten des Hundeführers beeinflussen das Verletzungsrisiko, insbesondere im Hinblick auf Mikrotraumen, deren Folgen erst später u.a. in Form von vermehrtem Verschleiß auftreten [4].

In einer Studie zum Thema Verletzungen beim Agility wurden über 1600 Fragebögen von Hundehaltern, die ihren Hund im Agility führen, ausgewertet [3]. Erfragt wurde, ob der jeweilige Hund in den vergangenen 2 Jahren im Rahmen des Agility-Trainings oder einer Turnierteilnahme Verletzungen erlitten hat. Differenziert wurde nach den verschiedenen Hunderassen sowie nach der Lokalisation der Verletzung. 33\% der Hunde erlitten in einem 2-Jahres-Zeitraum Verletzungen, die für den Hundehalter offensichtlich waren.

Border Collies, Australien Shepherds und Shelties erlitten überproportional häufig Verletzungen. Man mag versucht sein, dies damit zu erklären, dass diese Hunderassen im Agility auch überproportional häufig vertreten sind. Dies wird allerdings widerlegt, wenn man gleichzeitig die prozentuale Rasseverteilung der unverletzten Hunde betrachtet. Hier sind Border Collies und Shelties mit weit weniger Prozentpunkten vertreten als in der Population der Hunde mit Verletzungen. Von Verletzungen betroffen waren vorwiegend Schulter und Rücken. Verletzungen der Knie, Hüfte, Karpalgelenke und Zehen traten weit seltener auf. 
Die gesamte Studie umfasst allerdings lediglich Verletzungen, die für den Besitzer offensichtlich waren und in erkennbarem Zusammenhang mit dem Hundesport standen. Darüber hinaus kommt es bei Agility-Hunden fast immer zu Blockaden in den unterschiedlichsten Körperregionen. Häufig betroffen sind die Iliosakralgelenke, die Lenden- oder vordere Brustwirbelsäule, die Schulter-, Ellenbogen- und Zehengelenke. Oft führen diese Subluxationskomplexe irgendwann zu klinischen Symptomen, die mehr oder weniger deutlich in der Ausführung des Sportes oder auch im Alltag auffallen.

\section{Flyball}

Beim Flyball sollen die Hunde mit den Pfoten eine Ballwurfmaschine bzw. Attrappe betätigen, den Ball auffangen und wieder an die Startlinie bringen. Bei dieser Sportart geht es ebenfalls um ein hohes Tempo. Während zu Beginn des Trainings vor allem die Vorderextremitäten beansprucht werden, werden durch die steigende Routine der Hunde, die zunehmende Geschwindigkeit und die immer gleiche Ausführung der Aktion zahlreiche Regionen des Körpers in immer gleicher Weise beansprucht $[1,4]$. Die Karpalgelenke werden unabhängig vom Ausbildungsstand während der Betätigung der Ballwurfmaschine stets in starke Hyperextension gebracht. Diese einseitige Belastung kann sehr schnell zu Mikrotraumen und Blockaden in den unterschiedlichsten Körperregionen führen - fast immer im Schulter-, Ellenbogen- und Karpalgelenk.

\section{Obedience}

Hunde, die im Obedience gearbeitet werden, erlernen bereits in jungem Alter die „korrekte Fußarbeit“. Erwünscht ist, dass der Hund an der linken Seite des Hundeführers stets mit seinem Schulterblatt auf Kniehöhe des Menschen verbleibt und Blickkontakt zu seinem Menschen hält (ه Abb. 1). Dies führt zu einem stets nach oben und rechts gerichtetem Kopf und einer entsprechend gedrehten Halswirbelsäule [4]. Entsprechend dieser einseitigen Belastung der Halswirbelsäule kommt es hier zu Bewegungseinschränkungen in den Gelenken, insbesondere im Genick, aber auch in den Facettengelenken der einzelnen Halswirbel sowie zu einseitigen Muskelspannungen.

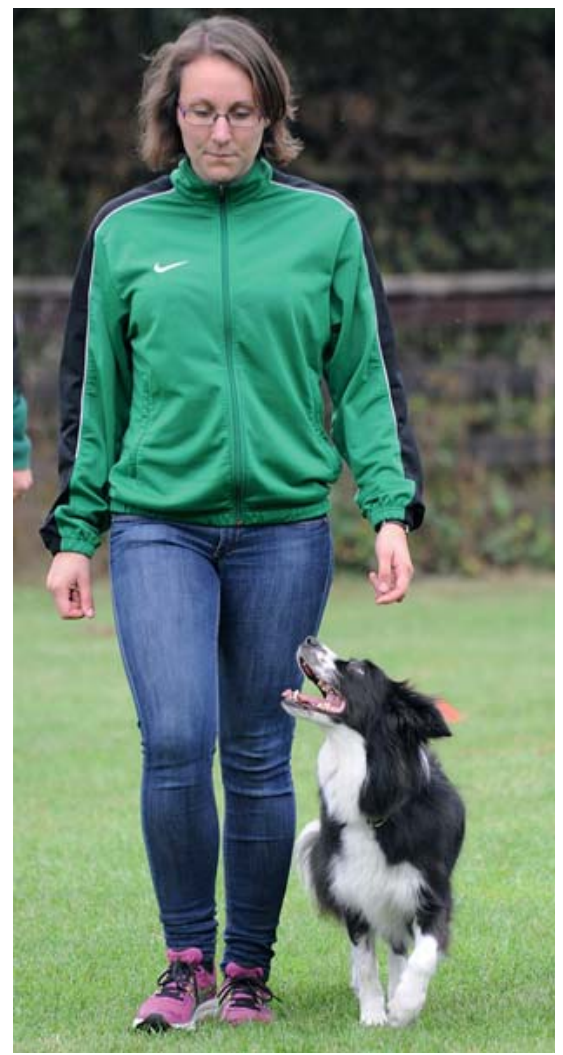

Abb. 1 Obedience-Hund bei der Fußarbeit. (C) Inga Finnberg

\section{Schutzhundsport}

Im Schutzhundsport werden die Hunde neben der gängigen Unterordnung u.a. auch im Schutzdienst ausgebildet. Sie lernen, potenzielle Angreifer zu stellen, zu verbellen und auf Kommando auch in eine vorgegebene Lokalisation - den sogenannten Beißarm - zu beißen. Diesen Beißarm sollen sie festhalten, obwohl der Träger den an seinem Arm hängenden Hund in die Luft hebt und körperlich bedrängt, den Arm wieder loszulassen. Während dieses Vorganges wird insbesondere die Halswirbelsäule der Hunde stark beansprucht. Die Folge sind häufig Blockaden in den Facettengelenken sowie im Genick.

\section{Sporthunde in der tierärzt- lichen Praxis für Chiropraktik}

Nach den Aufzeichnungen der Autorin aus dem eigenen Patientengut sind es häufig recht subtile Probleme, mit denen die sportlich geführten Patienten vorgestellt werden. Die Hunde zeigen Veränderungen im Bewegungsablauf, unterlaufen Hürden, schmeißen Stangen, mitunter nur bei bestimmten Sprunganforderungen, sie taxie- ren vor Sprüngen, verlieren an Tempo. Sie werden ungenauer in der Fußarbeit, verweigern den Beißarm oder lassen ihn vorzeitig los. Seltener sind es tatsächliche Lahmheiten, mit denen die Patienten vorgestellt werden. Dennoch lassen sich bei den Patienten in der Regel Veränderungen im Gangbild beobachten. Asymmetrische Bewegungen im Beckenbereich, eine wenig schwingende Rückenlinie, Rutenschiefhaltung, geringgradige Kopfschiefhaltungen, diagonaler Gang oder Passgang sind nur einige der möglichen Auffälligkeiten.

\section{So diagnostiziert der Chiropraktiker}

Die Diagnostik der Schulmedizin umfasst neben der Allgemeinuntersuchung die spezielle orthopädische und spezielle neurologische Untersuchung. Darüber hinaus gibt es im Rahmen der bildgebenden Verfahren die Möglichkeit, strukturelle Veränderungen an Knochen, Gelenken und Weichteilgewebe zu diagnostizieren. Häufig sind hier jedoch bei den in der chiropraktischen Praxis vorgestellten Patienten keinerlei Hinweise auf die Ursache des Problems zu finden.

An Therapiemöglichkeiten sieht die Schulmedizin in erster Linie Ruhe und medikamentöse Schmerz- und Entzündungshemmung vor [2]. Dies lindert oft - zumindest zeitweise - die Symptome. Nach Absetzen der Medikamente treten jedoch zumindest die subtileren Symptome meistens wieder auf. Für die Hundesportler ist der dauerhafte Einsatz von Medikamenten keine Lösung, wenn der Hund weiter in der jeweiligen Disziplin arbeiten soll.

Allgemeine und spezielle Untersuchung

Die Diagnostik eines Chiropraktikers sieht ebenfalls die Allgemeinuntersuchung sowie die spezielle orthopädische und neurologische Untersuchung vor. Es gilt, sicherzustellen, ob bei dem Patienten Probleme vorliegen, die eine primäre oder begleitende schulmedizinische Versorgung erfordern (z. B. Kreuzbandrisse).

\section{Ganganalyse}

Die Ganganalyse des Chiropraktikers umfasst neben den Aspekten, die auch in der Schulmedizin von Belang sind (Lahmheiten, Ataxien, Durchtrittigkeit, Fehlstellungen o. Ä.), noch weitere Feinheiten. Er 
achtet u.a. auf minimale ungleichmäßige Belastungen, wenig Schwingung in der Rückenlinie oder in einzelnen Arealen des Rückens, ungleichmäßige oder eingeschränkte Bewegung im Beckenbereich, mehr oder weniger ausgeprägte Steifheit im Gang in einzelnen Gliedmaßen bzw. einzelnen Gelenken.

\section{Palpation}

Bei der Palpation unterscheidet der Chiropraktiker die

- statische Palpation, bei der er u.a. auf Temperaturunterschiede der Haut, ungleichmäßig ausgebildete Muskulatur, Verhärtungen in der Muskulatur sowie anatomische Veränderungen achtet, und die

- Bewegungspalpation, bei der das Bewegungsausmaß der einzelnen Bewegungseinheiten (Gelenke) der Wirbelsäule und der Gliedmaßen untersucht wird.

\section{Chiropraktische Therapie}

Die Therapie des Chiropraktikers zielt auf die Beseitigung der Ursachen für die klinischen Symptome sowie auf die Wiederherstellung des inneren Gleichgewichts des Organismus ab. Der Chiropraktiker betrachtet - wie Angehörige vieler anderer nicht schulmedizinischer Professionen auch - den Patienten und seinen Organismus ganzheitlich. Mit den spezifischen Adjustments an den bewegungseingeschränkten Gelenken wird zum einen die normale Beweglichkeit wieder hergestellt, zum anderen werden die Selbstheilungskräfte des Körpers aktiviert, sodass der Organismus in sein ursprüngliches Gleichgewicht finden kann.

Die Chiropraktik ersetzt die klassische Veterinärmedizin nicht, bietet bei vielen Erkrankungen aber eine sinnvolle Ergänzung zur Rehabilitation und zur Gesunderhaltung.

\section{Fallbeispiele Border-Collie-Hündin mit diversen Blockaden}

Mia ist eine 5-jährige Border-Collie-Hündin. Sie wird im Obedience und Agility geführt. Zum Zeitpunkt der Erstvorstellung hatte die Hundebesitzerin die Hündin seit
3 Monaten aus dem Sportgeschehen herausgenommen. Mia hatte angefangen, vor dem Springen die Hürden zu taxieren, riss vermehrt die Stangen, hatte große Schwierigkeiten, bestimmte Anforderungen im Parcours zu bewerkstelligen. Ebenso zeigte sie im Alltag Probleme, spielte nicht mehr so häufig mit Ihren Artgenossen, tat sich schwer, auf ihren erhöhten Liegeplatz zu kommen, taxierte vor dem Sprung in den Kofferraum. Entstanden ist diese Problematik, nachdem Mia mehrmals unglücklich in Hindernisse hineingesprungen war.

Anfänglich hatte die Hundehalterin ihren Haustierarzt aufgesucht, der Hund bekam ein NSAID sowie eine Sportpause verordnet. Nachdem dies zu keinerlei Besserung des Problems führte, ließ die Besitzerin Röntgenbilder der Wirbelsäule und der Hüfte anfertigen. Hier waren keine strukturellen Veränderungen zu erkennen. Der Haustierarzt konnte nicht weiterhelfen.

Weil die Hundebesitzerin nicht aufgeben wollte, wurde die Hündin in der chiropraktischen Praxis vorgestellt. Hier zeigte die Hündin einen leicht steifen Gang in der Hinterhand, minimale Innenrotation des rechten Tarsalgelenkes, eine kaum vorhandene Beweglichkeit im Becken sowie eine wenig schwingende Rückenlinie. Palpatorisch waren die lange Sitzbeinmuskulatur sowie der M. quadriceps an der rechten Hinterhand geringgradig schlechter ausgebildet als auf der linken Seite.

In der weitergehenden chiropraktischen Untersuchung wurden insbesondere Bewegungseinschränkungen in beiden Iliosakralgelenken sowie der hinteren Lendenwirbel gefunden. Außerdem waren vertebrale Subluxationskomplexe zwischen T9 und T12 sowie an T4 und T5 und am Atlas zu finden. Darüber hinaus waren beidseits die Schultergelenke sowie die Ellenbogengelenke blockiert.

Bereits nach der ersten chiropraktischen Behandlung zeigte Mia eine deutliche klinische Besserung, ihr Verhalten im Alltag normalisierte sich, der Kofferraum stellte kein Hindernis mehr dar. Auch das Gangbild verbesserte sich, es war lediglich eine geringgradig asymmetrische Bewegung im Becken zu sehen. Nach einer weiteren chiropraktischen Behandlung 14 Tage später wurde zusätzlich mit gezielten Muskelaufbau-Übungen begonnen (Wackelbrett, isometrische Übungen, spe- zifisches Tricktraining), um die Muskulatur zu stärken und so den Gelenken eine ausreichende Stabilität zu geben.

Mit sportlichem Training wurde erst nach weiteren 4 Wochen, einer weiteren chiropraktischen Behandlung sowie vollständiger Normalisierung des Gangbildes begonnen. Der Trainingsaufbau wurde in Art und Intensität fachlich begleitet, und die Anforderungen für den Hund wurden nur sehr moderat gesteigert.

Dennoch konnte Mia die steigenden Anforderungen problemlos bewältigen und letztlich auch wieder auf Turnierniveau arbeiten. Bei den folgenden regelmäßigen chiropraktische Kontrollen in größer werdenden Abständen ergaben sich nur minimale Veränderungen an einzelnen Gelenken, die unmittelbar behandelt wurden, sodass Kompensationsblockaden vermieden werden konnten. Mia läuft mittlerweile wieder in der höchsten Agility- sowie in der höchsten Obedience-Klasse und wird lediglich 2-mal jährlich routinemäßig chiropraktisch durchgecheckt.

\section{Australian-Shephard-Hündin mit blockiertem Iliosakralgelenk}

Easy ist eine 2-jährige Australian-Shepherd-Hündin, die im A2, also der mittleren Leistungsklasse, im Agility geführt wird. Easy hatte ca. einen Monat vor der Vorstellung in der chiropraktischen Praxis einen Wespenstich im Zwischenzehenbereich an der linken Vordergliedmaße. Da die Hündin in der Folge zur Automutilation im Bereich des Wespenstichs neigte und ein Ekzem bekam, musste sie über 14 Tage mit einem Schutzschuh laufen.

Im Anschluss, nachdem die Hündin wieder ohne Fußschutz laufen durfte und schulmedizinisch als geheilt galt, fiel ein deutlich verändertes Gangbild auf. Die Hündin lief nicht mehr in einer geraden, sondern in links-diagonaler Spur. Das Becken schwang deutlich, fast übermäßig nach rechts, die Bewegung nach links war minimal. Die Rute wurde stets geringgradig nach links getragen. In Kreisbewegungen sah man deutlich, dass die Hündin bei der Linksdrehung Schwierigkeiten hatte. Dieses Problem fiel auch im Sport bei Sprüngen und Wendungen auf. Palpatorisch waren eine erhöhte Muskelspannung des M. quadriceps sowie eine Empfindlichkeit im Bereich der Psoasmuskulatur am rechten Hinterlauf auffällig. 

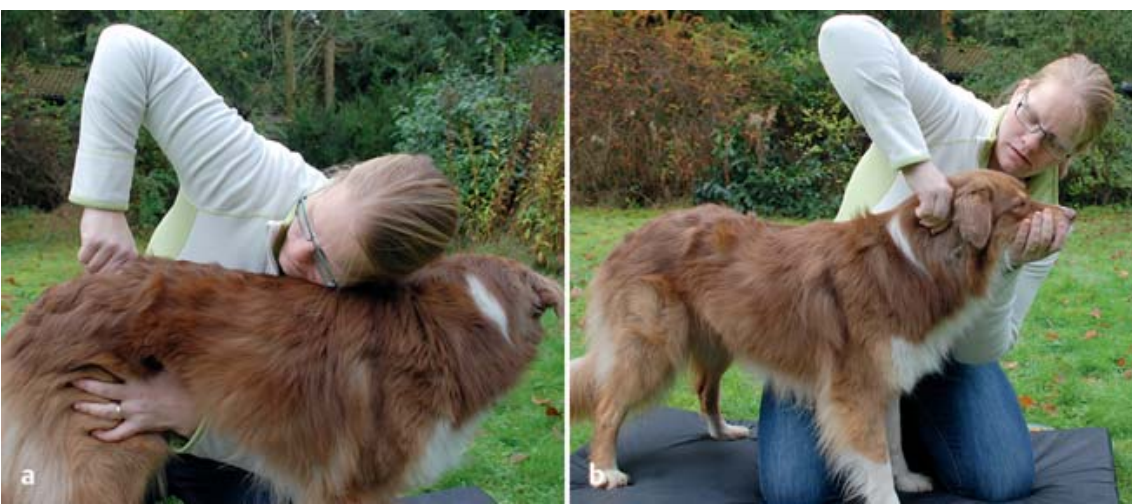

Abb. 2 a und b Chiropraktische Behandlung von Easy: a an der Lendenwirbelsäule, b am Atlas. (C) Andrea Reinhardt

Die chiropraktische Untersuchung ergab eine deutliche Bewegungseinschränkung im linken und eine etwas weniger ausgeprägte im rechten Iliosakralgelenk. Das Kreuzbein war nach links blockiert. Ebenso fanden sich vertebrale Subluxationskomplexe im kaudalen Bereich der Lendenwirbelsäule sowie in den vorderen Brustwirbeln (T3/4/7 und 9). Das rechte Schulterblatt sowie beide Ellenbogen waren fest. Bereits nach einmaliger chiropraktischer Behandlung ( $\$$ Abb.2) normalisierten sich das Gangbild sowie das Sprungverhalten vollständig. In einer chiropraktischen Kontrolle 3 Wochen später konnten keine nennenswerten Bewegungseinschränkungen mehr gefunden werden.

\section{Fazit}

Der Einsatz der Hunde im Sportbereich führt spartenspezifisch zu teilweise hohen und/oder einseitigen körperlichen Belastungen. Dabei kann es nicht nur zu offensichtlichen Verletzungen, sondern weitaus häufiger zu subtileren, zum Teil sogar unbemerkten Schädigungen am Bewegungsapparat kommen. Aufmerksame Hundeführer stellen Veränderungen in der Leistungsfähigkeit oder im Bewegungsablauf ihrer Vierbeiner frühzeitig fest. Oft sind dann in der schulmedizinischen Untersuchung inklusive der bildgebenden Verfahren keinerlei pathologische Befunde fest- zustellen. Als Tier-Chiropraktiker findet man bei diesen Patienten häufig deutliche Bewegungseinschränkungen in den Gelenken der Wirbelsäule oder/und der Gliedmaßen. Diese Bewegungseinschränkungen führen unbehandelt auf Dauer zu weiteren strukturellen Veränderungen (z.B. Muskelverhärtungen, Muskelasymmetrien, Spondylarthrosen, Arthrosen und andere Verschleißerscheinungen). Die chiropraktische Untersuchung des Sporthundes kann helfen, diese Folgeerscheinungen $\mathrm{zu}$ vermeiden und die ursprüngliche Leistungsfähigkeit wieder herzustellen. Optimalerweise werden sportlich geführte Hunde in regelmäßigen (individuell festzulegenden) Intervallen prophylaktisch chiropraktisch untersucht und behandelt. $\odot$

\section{Summary}

\section{Chiropratic in sports dogs:}

Effects of agility, obedience \& Co

The use of dogs in sport leads category specific to partially high and/or one-sided physical strain. This can lead not only to obvious injuries but more often to subtle and sometimes even unnoticed damage to the musculoskeletal system. Attentive dog handlers can notice changes in the performance or in the movement of their four-legged friends at an early stage. Often there are no pathological findings in medical examination, even when including imaging techniques. As an animal chiropractor, significant movement restrictions in the joints of the spine and/or the limbs can often be found in these patients. These movement restrictions can lead to further structural changes (e.g. muscle tightness, muscle asymmetry, spondylarthritis, osteoarthritis and other deterioration) if continually left untreated. The chiropractic examination of the sport dog can help to avoid these after-effects and restore the former performance. Optimally, dogs used in sport should be chiropractically examined at regular intervals (individually determined) and treated prophylactically.

(- Key words

chiropractic - dog - agility - flyball - obedience

\section{() Literatur}

[1] Baltzer W. Which injuries are most common in various sports? Im Internet: http:// veterinarymedicine.dvm360.com/ which-injuries-are-most-common-varioussports?rel=canonical; Stand 13.10.2014

[2] Hellebrekers LJ. Schmerz und Schmerztherapie beim Tier. Hannover: Schlütersche; 2001

[3] Levy M, Hall ChB, Trentacosta $\mathrm{N}$ et al. A survey of injuries occurring in dogs participating in agility. Vet Comp Orthop Traumatol 2009; 22: 321-324

[4] Robertson J, Mead A. Physical Therapy and Massage for the Dog. London: Manson Publishing; 2013

[5] Roth A, Tschanz-Haas R. Agility - Vom Junghund zur Leistungsklasse. Stuttgart: Müller Rüschlikon bei Paul Pietsch; 2012

\section{Online zu finden unter}

http://dx.doi.org/10.1055/s-0034-1383425

\section{? Johanna Reinhardt \\ Tierärztin \\ IVCA-zertifizierte Tier-Chiropraktikerin \\ Consrader Str. 29 \\ 19086 Consrade \\ E-Mail: info@chiro-fuer-tiere.de \\ www.chiro-fuer-tiere.de}

Approbation 2008; Tätigkeit in einer Kleintierklinik 2008-2013; Weiterbildung in Veterinärchiropraktik an der IAVC 2011; Zertifizierung der International Veterinary Chiropractic Association seit 2011; selbstständig in eigener Praxis mit Schwerpunkt Chiropraktik seit 2014 